\section{Questions les plus fréquentes sur le TarMed}

Après l'introduction du TarMed, pourrai-je, en tant que médecin au bénéfice d'une "formation postgraduée de deux ans selon la LAMal" facturer des prestations avec les caisses-maladie? Quelle valeur intrinsèque qualitative m'attribuera-t-on?

Vous ne pourrez facturer que des prestations de base générales et des prestations qui peuvent être facturées avec elles (désignées dans la structure tarifaire par pro memoria).

En tant que médecin praticien sans titre FMH, j'exerce principalement dans le domaine de la psychiatrie et psychothérapie en cabinet médical privé. A part ça, j'ai également une activité restreinte de généraliste (mon ancien métier) en médecine de premier recours (5 à $10 \%$ ). J'ai l'intention d'accomplir ma formation continue obligatoire en psychiatrie et psychothérapie. Selon le TarMed, pourrai-je continuer d'exercer ce petit pourcentage de prestations de base SANS formation continue complémentaire en médecine de premier recours?

H. G. $\dot{a} B$.

Non. Chaque activité médicale demande une formation continue régulière pour l'exercer.

Pos. TarMed 04.0040: que signifie ici "par série de tests»?

J. M. ’̀ W.

Cela signifie que la rémunération évoquée vaut pour le nombre d'échantillons prévu dans la série de tests, autrement dit pas pour chaque échantillon.

1. Pour les examens de cardiologie pédiatrique, il faut souvent pratiquer une oxymétrie, position que je n'ai pas trouvée dans le TarMed! Veuillez m'expliquer cet oubli.

2. Comment facture-t-on une oxymétrie en tant que prestation isolée lors d'une consultation en cardiologie?

P. H. $\dot{a} B$.

ad 1: La proposition a été déposée pour la révision 2000.

ad 2: Je ne peux répondre actuellement à cette question, qui sera traitée au cours de la révision 2000.
Dans le TarMed, le lieu où la prestation est apportée est désignée par "a1" ambulatoire, "a2" le plus souvent hospitalier et "S" hospitalier. Certaines prestations désignées par la lettre s sont aujourd'hui également fournies ambulatoirement. La désignation "S" signifie-t-elle que les prestations qui s'y rattachent ne peuvent être facturées que par un hôpital, même si l'examen est pratiqué en ambulatoire (ce qui réintroduirait le tarif orange ou Catalogue des prestations hospitalières) ou que ces prestations ne peuvent plus être facturées en ambulatoire (même pas par les hôpitaux), impliquant toujours une (courte) hospitalisation?

H. V. $\dot{a} Z$.

Les prestations «S" peuvent être facturées en ambulatoire. Cela a été confirmé par les assureurs qui voulaient pouvoir effectuer un transfert vers le secteur ambulatoire. Il s'agirait de citer les prestations en question afin de pouvoir les déplacer en «a2».

En tant que rhumatologue (autrefois FMH en médecine physique), j'ai beaucoup investi dans le domaine physiothérapeutique. Je n'arrive pas à comprendre pourquoi la FMH a exclu aussi simplement la physiothérapie du GRAT. Les domaines du laboratoire et de la radiologie ne sont pas non plus des prestations directement médicales. A l'instar de la radiologie effectuée par une assistante médicale, mes physiothérapeutes font de la physiothérapie selon mes instructions spéciales. Il s'agit souvent de cas complexes qui ont été traités auparavant par des médecins généralistes. La seule alternative serait une coûteuse hospitalisation et un traitement hospitalier. Il semble, selon mes dernières informations, que les tarifs Reha promis ne seront réservés qu'à des cas spéciaux encore plus coûteux en clinique de réadaptation. En comparaison, c'est comme si l'on enlevait le scalpel à un chirurgien. Devons-nous vraiment facturer au tarif de physiothérapie ridiculement bas et nous laisser conduire à la ruine? Pouvons-nous, somme toute, facturer au tarif de physiothérapie sans être partenaires contractuels? Ou peut-on nous retirer ce droit sans conditions? La FMH ne devait-elle pas au moins régler par convention les positions que nous devons utiliser en tant que médecins selon les situations pour des cas complexes (pos. 1073)? Ou doit-on en déduire que l'on laisse tomber tout un groupe de spécialistes?

\section{A. W. $\dot{a} W$.}

La physiothérapie n'a jamais été contenue dans le GRAT, elle ne pouvait donc pas en être exclue. Les prestations facturables par le cabinet médical seront répertoriées dans le tarif de physiothérapie. Les prestations complexes de physiothérapie attendent leur tarification, sous le mot de code Tar-Rehab. 
Le bruit court que la FMH veut que la tarification de l'examen radiologique au cabinet médical du généraliste (maintien de la position pour l'enclenchement et le déclenchement de l'appareil) soit plus élevé que celle de l'examen pratiqué par le spécialiste FMH en radiologie, ceci même après la révision du tarif en radiologie. Est-ce-vrai?

R. B. $\dot{a} B$.

Ne vous fiez pas aux bruits qui courent. Il suffit de jeter un coup d'œil à la version alpha 2.2. Si la rumeur se confirme, ce ne sera pas sous la forme exagérée que vous lui donnez. Partant de la décision de principe selon laquelle le cabinet de médecine de premier recours doit également pouvoir offrir des prestations en radiologie native et les facturer, une prestation plancher devait être calculée pour ce type de cabinet sans laquelle les coûts des radiographies ne pourraient être couverts (cf. degré d'exploitation).

Il s'agit d'une décision de principe, de nature professionnelle et politique, qui devait être transposée dans le tarif. Si vous avez d'autres remarques à faire, vous voudrez bien vous adresser à qui de droit.

Chaque soir, après mes consultations, j'examine les dossiers de patients du lendemain et me prépare de manière ciblée, ce qui prend entre $20 \mathrm{sec}$. et $10 \mathrm{~min}$. par patient. Je consacre chaque soir entre 30 à 40 min. à ce travail, cinq fois par semaine. Cette activité sera-t-elle rémunérée dans le TarMed?

\section{J. R. $\dot{a} I$.}

Cette activité est, le cas échéant, une partie anticipée de la consultation, mais dans une certaine mesure seulement. Le travail pour le patient en son absence ne peut être facturé ici tant qu'il s'agit de l'étude de ses propres documents.
En tant que spécialiste en médecine générale et mère de famille, je travaille depuis quatre ans à temps partiel et presque exclusivement en gynécologie. Je prends part au service d'urgence avec les médecins de premier recours et suit le programme de formation continue de la SSMG. Ma formation continue est centrée sur la médecine générale et la gynécologie.

Dans le TarMed, les positions "premier examen gynécologique, consultation gynécologique (pilule contraceptive, hormonothérapie, etc.) et examen de grossesse" sont attribuées, selon la valeur intrinsèque qualitative, aux spécialistes en gynécologie et obstétrique.

Mes questions sont les suivantes:

1. Pourrai-je continuer à facturer dans mon cabinet ces positions qui sont pour moi existentielles (principe des droits acquis)?

2. Quelle est l'instance qui décidera, en cas d'espèce, si la prestation a été "régulièrement apportée et jusqu'ici sans contestation»?

3. Est-il vrai que les examens préventifs et les conseils ne pourront plus être facturés par les médecins de premier recours (alors que par ex. les positions "examen au spéculum" et "colposcopie" demeureront ouvertes à tous)?

C. L. $\dot{a} F$.

ad 1: $\quad$ Oui.

ad 2: La FMH, puis la nouvelle organisation TarMed.

ad 3: Je ne peux répondre à la question sans en savoir plus. Certains de ces examens dépendent en effet de définitions et réglementations relevant du droit fédéral qui ne peuvent bien entendu être modifiées par les parties contractantes. 